\title{
Spontaneous light emission in complex nanostructures
}

\author{
L. A. Blanco ${ }^{1}$ and F. J. García de Abajo ${ }^{1,2}$ \\ ${ }^{1}$ Donostia International Physics Center (DIPC), Apartado Postal 1072, 20080 San Sebastián, Spain \\ ${ }^{2}$ Centro Mixto CSIC-UPV/EHU, Aptardo Postal 1072, 20080 San Sebastián, Spain
}

(Received 29 August 2003; revised manuscript received 17 March 2004; published 28 May 2004)

\begin{abstract}
The spontaneous emission of an excited atom surrounded by different materials is studied in the framework of a semiclassical approach, where the transition dipole moment acts as the source of the emission field. The emission in the presence of semiinfinite media, metallic nanorings, spheres, gratings, and other complex geometries is investigated. Strong emission enhancement effects are obtained in some of these geometries associated to the excitation of plasmons (e.g., in nanorings or spheres). Furthermore, the emission is shown to take place only along narrow angular distributions when the atom is located inside a low-index dielectric and near its planar surface, or when metallic nanogratings are employed at certain resonant wave lengths. In particular, axially symmetric gratings made of real silver metal are considered, and both emission rate enhancement and focused far-field emission are achieved simultaneously when the grating is decorated with further nanostructures.
\end{abstract}

DOI: 10.1103/PhysRevB.69.205414 PACS number(s): 78.55.-m, 42.25.Fx, 42.79.Dj, 78.67.Bf

\section{INTRODUCTION}

The spontaneous emission (SE) of photons by excited atoms has captured the attention of many researchers for a long time. ${ }^{1-12}$ When an excited atom is placed in a homogeneous medium, the emitted electromagnetic field takes the same form as that produced by an electric dipole. The directional intensity of the dipolar far field exhibits a maximum value for the direction perpendicular to the dipole, while the emission is forbidden along the parallel direction. ${ }^{13}$

Nevertheless, the SE field can be strongly modified if the atom is surrounded by materials of different composition and shape, as it was predicted by Purcell ${ }^{1}$ and later corroborated experimentally. ${ }^{14-16}$ Theoretical proposals along this direction have been made, some of them consisting in surrounding the emitter by different kinds of dielectrics and metals. ${ }^{2,17-19}$

In particular, photonic crystals have been used to control light propagation ${ }^{3-6,20-25}$ and to achieve enhancement $^{5,6,22,24,26}$ or suppression ${ }^{3}$ of the SE field. Another possibility consists in placing the atom inside an optical cavity: ${ }^{7,27-30}$ again this can lead to either inhibition ${ }^{8}$ or enhancement ${ }^{26}$ of the emission probability. Left-handed materials, ${ }^{9}$ mirrors, ${ }^{31-33}$ sharp metallic tips,${ }^{34}$ waveguides, ${ }^{35}$ or quantum $\operatorname{dots}^{36,37}$ can also be used to modify the SE features. The physical processes involved in these phenomena are of quantum nature, such as quantum interference, ${ }^{22}$ quantum degeneration, ${ }^{38}$ or proximity effects in plasmon resonances. ${ }^{10}$

The modification of SE by a dielectric sphere has been studied analytically within the framework of classical ${ }^{9,39}$ and quantum ${ }^{40}$ electrodynamics, and also in the limit of strong interactions between the atom and the sphere, ${ }^{41}$ in which case the level populations experience Rabi oscillations and the decay rate is no longer a valid magnitude to describe the decay of the system. Rabi oscillations are important when the atom is placed inside a dielectric sphere, ${ }^{11}$ sometimes giving rise to enhancement of the SE. ${ }^{42-45}$

Metallic nanostructures have been shown to increase the strength of the electromagnetic field in the context of surface-enhanced Raman spectroscopy (SERS), ${ }^{46-50}$ in a similar way as in SE.

Not only the emission probability is modified by the environment, but the angular distribution of emitted photons can be substantially changed and in some cases the SE field shows a very sharp directionality. ${ }^{33,36}$ This is the case in particular of metallic wave guides with some additional structure. ${ }^{51,52}$ This type of geometries has been applied to microwaves since the 1970's to increase the directionality of oriented antenna designs, and renewed interest has emerged more recently as applications in the visible and near infrared regions are becoming practical. ${ }^{53-57}$ More specifically, light can be funneled into hole arrays much smaller than the wavelength ${ }^{53}$ and also through subwavelength slits in metallic thin films. ${ }^{56}$ Light collimation by a hole decorated on the exit side of the film, ${ }^{54,55}$ and enhancement in transmission by decorating the input side ${ }^{54,57}$ have also been achieved. Directionality in antenna designs can be also obtained by modifying the properties of the ground plane. ${ }^{58}$

In this work, we present a simple model of SE in semiinfinite media as a first example of systems that can give rise to both enhancement and directionality effects. The emitting atom is described by the electric dipole moment corresponding to the atomic transition under consideration.

More elaborated geometries need other sophisticated tools which permit solving Maxwell's equations in the presence of objects of arbitrary shape. Among them, both the Green's tensor method ${ }^{59-61}$ and the boundary element method (BEM),${ }^{62}$ have been successfully used to several problems; the latter will be the one applied in this work. The atom is described within a semiclassical approach and the boundary conditions on the interfaces between different materials are imposed by means of surface charge and current distributions, which will become the sources of the reflected and scattered electromagnetic fields. In the problem that concerns us, the BEM is applied to the study of the interaction of the SE field with spheres, rings, and gratings. Strong enhancement both in the total emission and in the angular direction- 
ality of the emitted field is achieved.

This paper is organized as follows: In Sec. II we present a semiclassical treatment of the SE process and apply it to the calculation of the emission width in a homogeneous medium; Sec. III is devoted a model for semi-infinite media and some results of its application are presented; A method for dealing with more complicated geometries will be the subject of Sec. IV, which will be applied to several metallic systems in subsequent sections (rings in Sec. VI, spheres in Sec. V, and gratings in Sec. VII). Finally, the main conclusions will be summarized in Sec. VIII.

\section{SPONTANEOUS EMISSION IN A HOMOGENEOUS MEDIUM}

We consider a two-level atom with $\Psi_{g}(\mathbf{r})$ and $\Psi_{e}(\mathbf{r})$ being the wave functions of the ground state and the excited state, respectively. With these wave functions we can build a SE electron current density ${ }^{63,64}$

$$
\mathbf{j}(\mathbf{r})=-\mathrm{i} \frac{e \hbar}{m} \Psi_{g}^{*}(\mathbf{r}) \vec{\nabla} \Psi_{e}(\mathbf{r})
$$

which, invoking the charge continuity equation, gives rise to a charge density given by

$$
\rho(\mathbf{r})=\frac{1}{\mathrm{i} \omega} \vec{\nabla} \cdot \mathbf{j}(\mathbf{r})
$$

A time dependence $e^{-i \omega t}$ is implicitly understood in this expression, where $\omega$ is the frequency of the emitted photon.

The electric field created by these densities can be written as

$$
\mathbf{E}(\mathbf{r})=\int d \mathbf{r}^{\prime}\left[\frac{\mathrm{i} k}{c} G\left(\mathbf{r}-\mathbf{r}^{\prime}\right) \mathbf{j}\left(\mathbf{r}^{\prime}\right)-\frac{1}{\epsilon} \vec{\nabla}_{\mathbf{r}} G\left(\mathbf{r}-\mathbf{r}^{\prime}\right) \rho\left(\mathbf{r}^{\prime}\right)\right]
$$

where $G(\mathbf{r})=\exp \left(\mathrm{i} k^{\prime} r\right) / r$ is the Green function of the scalar Helmholtz equation, $k=\omega / c$, and $k^{\prime}=k \sqrt{\epsilon}$.

Now, we make use of the dipole approximation, that is, the fact that the atomic dimensions are much smaller than the radiation wave length, and therefore we approximate

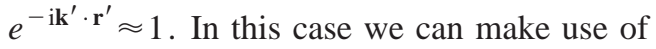

$$
\int d \mathbf{r j}(\mathbf{r})=-\mathrm{i} \omega \mathbf{d}
$$

where $\mathbf{d}=e\left\langle\Psi_{g}|\mathbf{r}| \Psi_{e}\right\rangle$ is the dipole matrix element of the electronic transition.

The part containing the charge density in Eq. (2) gives a longitudinal contribution to the far field, and since the total field has to be transversal, this longitudinal contribution must cancel out the one coming from the current density. In the far-field limit, the integral in Eq. (2) can be expressed in terms of the dipole matrix element $\mathbf{d}$ as

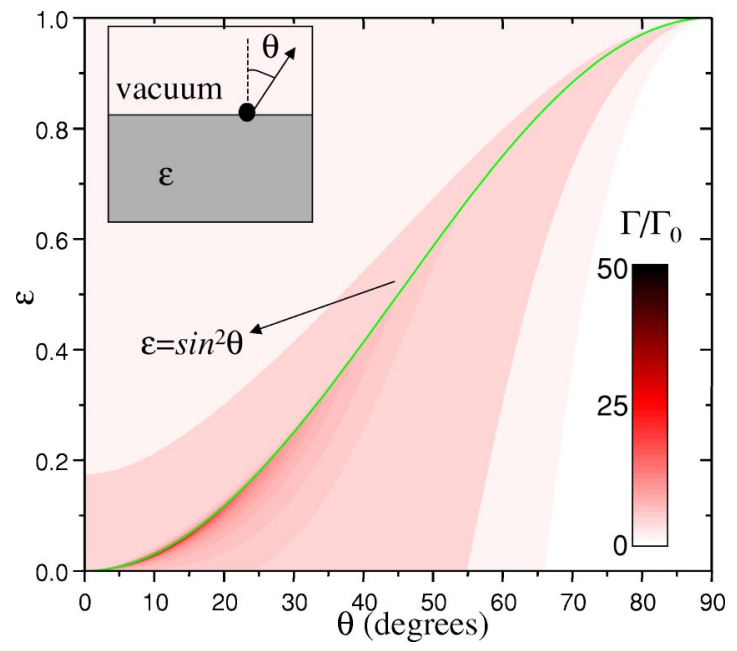

FIG. 1. (Color online) Spontaneous emission probability normalized to vacuum as a function of the angle of emission (see inset) for an atom placed at the surface of a semi-infinite medium of dielectric constant $\epsilon$ with vacuum. The maximum emission occurs along the curve $\epsilon=\sin ^{2} \theta$.

$$
\mathbf{E}(\mathbf{r} \rightarrow \infty)=\frac{e^{\mathrm{i} k^{\prime} r}}{r} \mathbf{f}
$$

where $\mathbf{f}=k^{2}[\mathbf{d}-\hat{\mathbf{r}}(\mathbf{d} \cdot \hat{\mathbf{r}})]$ is the far-field SE amplitude and $\hat{\mathbf{r}}$ $=\mathbf{r} / r$.

To calculate the emission probability per unit time, we construct the Poynting vector $\mathbf{S}$ from the electric and magnetic far fields as $\mathbf{S}=2 \operatorname{Re}\left[(c / 4 \pi)\left(\mathbf{E} \times \mathbf{H}^{*}\right)\right]$. Again in the far-field limit, $\mathbf{S} \cdot \hat{\mathbf{r}}=(c / 2 \pi)|\mathbf{f}|^{2} r^{-2} \sqrt{\boldsymbol{\epsilon}}$ and we find the emission rate to be

$$
\Gamma=\frac{1}{\hbar \omega} \int d \Omega r^{2} \mathbf{S} \cdot \hat{\mathbf{r}}=\frac{4}{3 \hbar} k^{3} \sqrt{\epsilon}|\mathbf{d}|^{2}
$$

\section{SPONTANEOUS EMISSION IN A SEMI-INFINITE DIELECTRIC MEDIUM}

One of the simplest systems in which SE can be studied is the one formed by semi-infinite planar media. ${ }^{31,33,65,66}$ Unlike in some other models, ${ }^{66}$ we do not consider the effect of radiative damping in the oscillating electric dipole. We will calculate the emission rate for only one atom with its transition dipole moment oriented along prescribed directions, from which averaging over dipole moment orientations can be trivially performed. We will apply a formalism similar to the one in Sec. II to investigate enhancement and directionality effects. For that purpose, we will place the atom in a medium with dielectric constant $\epsilon$ and study the propagation of radiation towards the surface that separates this medium $(z<0)$ from the vacuum $(z>0)$ (see insets of Figs. 1, 2, and 3).

\section{A. Spontaneous emission field}

Let us start from Eq. (2), taking into account the twodimensional translational symmetry of the surface that per- 


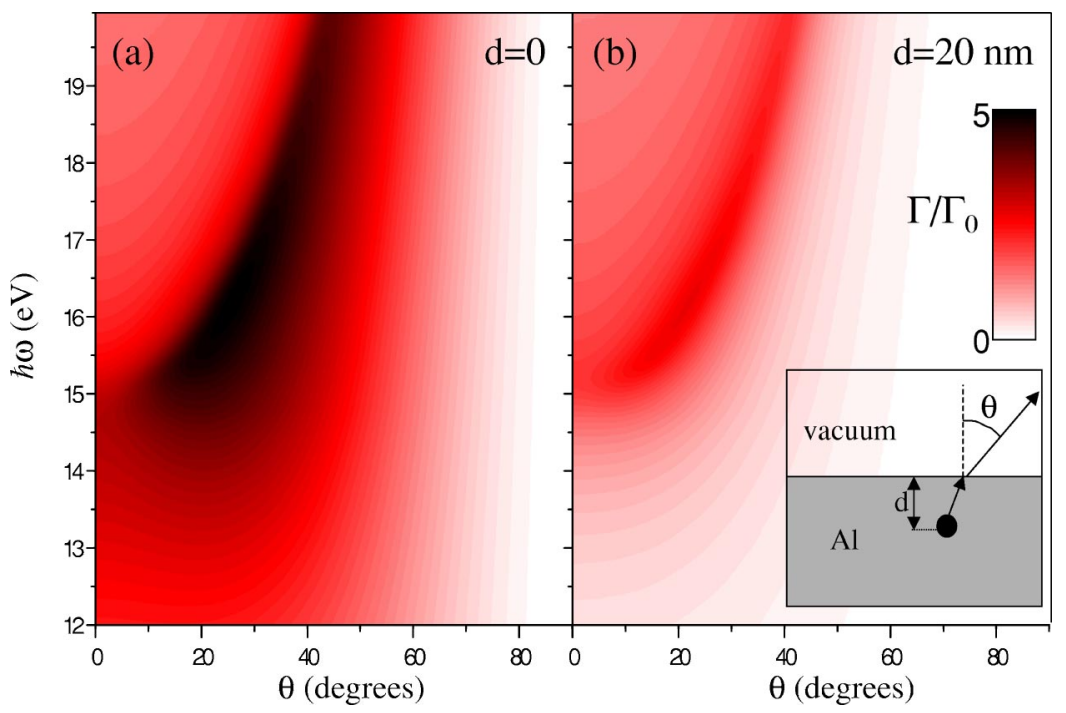

FIG. 2. (Color online) (a) Spontaneous emission probability normalized to vacuum for an atom placed at a planar Al-vacuum interface, as a function of polar angle of emission $\theta$ and photon energy $\hbar \omega$. (b) The same as (a) but when the atom is placed inside $\mathrm{Al}$ at a distance of $20 \mathrm{~nm}$ from the surface of separation between media.

mits separating the photon momentum $\mathbf{q}$ in a twodimensional part $\mathbf{Q}$ parallel to the plane $z=0$, and its perpendicular component $q_{z}$. We can express the Green function inside the medium described by $\epsilon$ as

$$
\begin{aligned}
G(\mathbf{r}) & =\frac{e^{\mathrm{i} k^{\prime} r}}{r}=\int \frac{d \mathbf{q}}{(2 \pi)^{3}} \frac{4 \pi}{q^{2}-k^{\prime 2}-\mathrm{i} 0^{+}} e^{\mathrm{i} \mathbf{q} \cdot \mathbf{r}} \\
& =\int \frac{d \mathbf{Q}}{(2 \pi)^{2}} \frac{2 \pi}{\Delta^{\prime}} e^{-\Delta^{\prime}|z|} e^{\mathrm{i} \mathbf{Q} \cdot \mathbf{R}},
\end{aligned}
$$

where $\mathbf{Q}=\left(Q_{x}, Q_{y}\right), \mathbf{R}=(x, y), \Delta^{\prime}=\sqrt{Q^{2}-k^{\prime 2}-\mathrm{i} 0^{+}}$, and $0^{+}$is an infinitesimally small positive real number.

Inserting Eq. (5) into Eq. (2) and noticing that one can replace $\vec{\nabla}_{\mathbf{r}}$ by $\left[i \mathbf{Q},-\Delta^{\prime} \operatorname{sign}\left(z-z^{\prime}\right)\right]$ inside the integrand of Eq. (2), one obtains

$$
\begin{aligned}
\mathbf{E}(\mathbf{r})= & \iint d \mathbf{r}^{\prime} \frac{d \mathbf{Q}}{2 \pi \Delta^{\prime}} e^{\mathrm{i} \mathbf{Q} \cdot\left(\mathbf{R}-\mathbf{R}^{\prime}\right)-\Delta^{\prime}\left|z-z^{\prime}\right|}\left[\frac{\mathrm{i} k}{c} \mathbf{j}\left(\mathbf{r}^{\prime}\right)\right. \\
& -\frac{1}{\mathrm{i} \omega \epsilon}\left[i \mathbf{Q},-\Delta^{\prime} \operatorname{sign}\left(z-z^{\prime}\right)\right] \\
& \left.\times\left\{\mathbf{j}\left(\mathbf{r}^{\prime}\right) \cdot\left[i \mathbf{Q},-\Delta^{\prime} \operatorname{sign}\left(z-z^{\prime}\right)\right]\right\}\right],
\end{aligned}
$$

where $\rho$ has been expressed in terms of $\mathbf{j}$ using Eq. (1), and integration by parts has been performed.

Making use again of the dipole approximation and of Eq. (3), we have

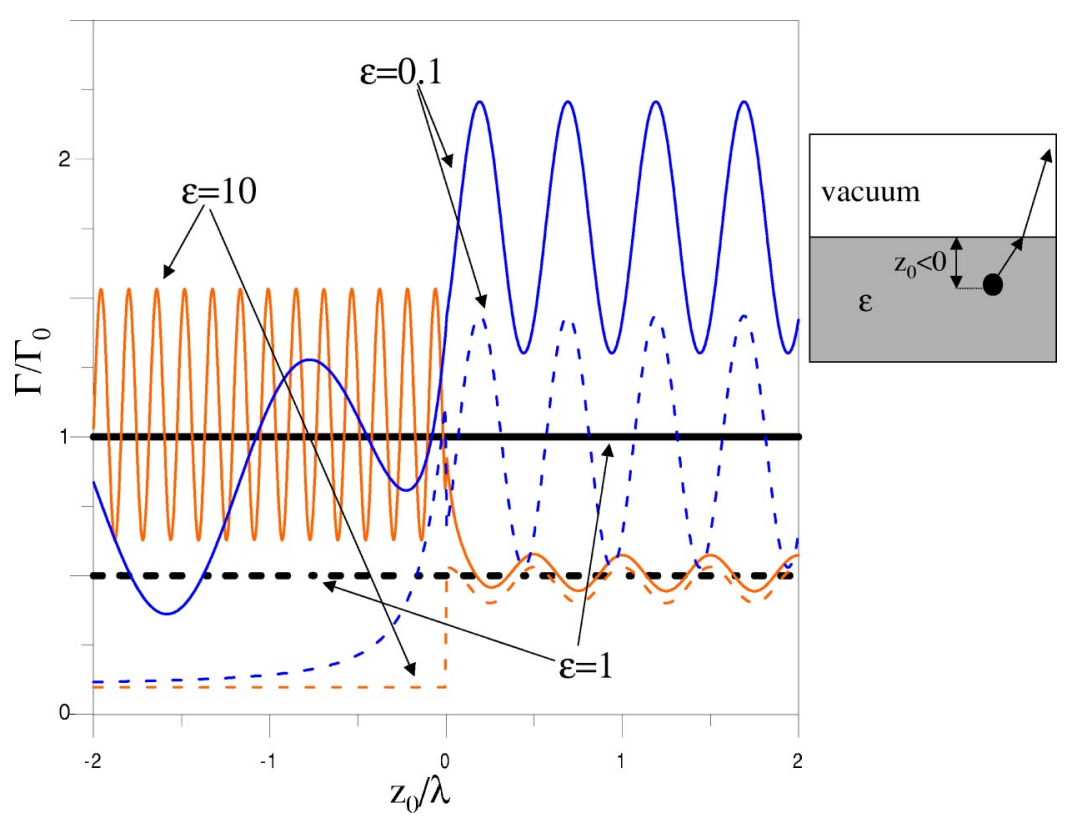

FIG. 3. (Color online) Spontaneous emission rate normalized to vacuum from an atom placed near a semi-infinite medium of dielectric constant $\epsilon$ for different positions of the atom (see inset). Dashed curves show the emission towards the vacuum side, while solid lines represent the sum of the emission towards both sides (vacuum and dielectric, i.e., the total emission). The atom lies inside the dielectric (vacuum) for $z_{0}<0 \quad\left(z_{0}\right.$ $>0)$. 


$$
\begin{aligned}
\mathbf{E}(\mathbf{r})= & \int \frac{d \mathbf{Q}}{2 \pi \Delta^{\prime}} e^{\mathrm{i} \mathbf{Q} \cdot \mathbf{R}-\Delta^{\prime}\left(z-z_{0}\right)} \\
& \times\left[k^{2} \mathbf{d}+\frac{1}{\epsilon}\left(\mathrm{i} \mathbf{Q},-\Delta^{\prime}\right) \mathbf{d} \cdot\left(\mathrm{i} \mathbf{Q},-\Delta^{\prime}\right)\right]
\end{aligned}
$$

Now, the field can be conveniently expressed in terms of $s$ and $p$ polarization vectors, $\mathbf{e}_{s}=(1 / Q)\left(-Q_{y}, Q_{x}, 0\right)$ and $\mathbf{e}_{p}$ $=\left(\mathrm{i} / k^{\prime} Q\right)\left(\Delta^{\prime} Q_{x}, \Delta^{\prime} Q_{y}, \mathrm{i} Q^{2}\right)$, as ${ }^{67}$

$$
\mathbf{E}(\mathbf{r})=\int d \mathbf{Q}\left(E_{s} \mathbf{e}_{s}+E_{p} \mathbf{e}_{s}\right)
$$

where

$$
\begin{aligned}
E_{s} & =\frac{1}{2 \pi \Delta^{\prime}} e^{\mathrm{i} \mathbf{Q} \cdot \mathbf{R}-\Delta^{\prime}\left(z-z_{0}\right)} \frac{k^{2}}{Q}\left(-d_{x} Q_{y}+d_{y} Q_{x}\right), \\
E_{p}= & \frac{1}{2 \pi \Delta^{\prime}} e^{\mathrm{i} \mathbf{Q} \cdot \mathbf{R}-\Delta^{\prime}\left(z-z_{0}\right)} \frac{\mathrm{i} k}{Q \sqrt{\epsilon}}\left(\Delta^{\prime} d_{x} Q_{x}+\Delta^{\prime} d_{y} Q_{y}\right. \\
& \left.+\mathrm{i} Q^{2} d_{z}\right)
\end{aligned}
$$

\section{B. Far field in vacuum with the emitting atom inside a dielectric}

The Snell law guarantees conservation of $Q_{x}$ and $Q_{y}$ at both sides of the surface, and only $q_{z}$ changes. The transmitted field components in vacuum are

$$
\begin{aligned}
& E_{s}^{t}=t_{s} E_{s}, \\
& E_{p}^{t}=t_{p} E_{p},
\end{aligned}
$$

where $t_{s}$ and $t_{p}$ are Fresnel's transmission coefficients. ${ }^{13}$

Details of the integration over $\mathbf{Q}$ in the $r \rightarrow \infty$ limit are given in Appendix A. The result for the Cartesian components of the far field in vacuum is the following:

$$
\begin{aligned}
& E_{x}=k^{2} \frac{e^{\mathrm{i} k r}}{r}\left(\frac{t_{s}}{\sqrt{\epsilon-\sin ^{2} \theta}} \cos \theta \sin \varphi a\right. \\
& \left.+\frac{t_{p} \cos \varphi}{\sqrt{\epsilon}} \frac{\cos ^{2} \theta}{\sqrt{\epsilon-\sin ^{2} \theta}} b\right) e^{-k \sqrt{\sin ^{2} \theta-\epsilon}\left|z_{0}\right|}, \\
& E_{y}=k^{2} \frac{e^{\mathrm{i} k r}}{r}\left(\frac{t_{s}}{\sqrt{\epsilon-\sin ^{2} \theta}} \cos \theta \cos \varphi(-a)\right. \\
& \left.+\frac{t_{p} \sin \varphi}{\sqrt{\epsilon}} \frac{\cos ^{2} \theta}{\sqrt{\epsilon-\sin ^{2} \theta}} b\right) e^{-k \sqrt{\sin ^{2} \theta-\epsilon}\left|z_{0}\right|}, \\
& E_{z}=k^{2} \frac{e^{\mathrm{i} k r}}{r}\left(-\frac{t_{p}}{\sqrt{\epsilon}} \frac{\sin \theta \cos \theta}{\sqrt{\epsilon-\sin ^{2} \theta}} b\right) e^{-k \sqrt{\sin ^{2} \theta-\epsilon}\left|z_{0}\right|}
\end{aligned}
$$

where the transmission coefficients can be expressed in terms of the emission angle $\theta$ as

$$
\begin{gathered}
t_{s}=\frac{2 \sqrt{\epsilon-\sin ^{2} \theta}}{\sqrt{\epsilon-\sin ^{2} \theta}+\cos \theta}, \\
t_{p}=\frac{2 \sqrt{\epsilon-\sin ^{2} \theta} \sqrt{\epsilon}}{\sqrt{\epsilon-\sin ^{2} \theta}+\epsilon \cos \theta},
\end{gathered}
$$

and

$$
\begin{gathered}
a=d_{x} \sin \varphi-d_{y} \cos \theta, \\
b=\sqrt{\epsilon-\sin ^{2} \theta}\left(d_{x} \cos \theta+d_{y} \sin \theta\right)-d_{z} \sin \theta .
\end{gathered}
$$

Finally, using the above expressions to calculate $|\mathbf{f}|^{2}$, the polar-angle dependent distribution of the transition rate is found to be

$$
\begin{aligned}
\frac{\Gamma(\epsilon, \theta)}{\Gamma_{0}}= & 2 \cos ^{2} \theta\left(\frac{1}{\left|\sqrt{\epsilon-\sin ^{2} \theta}+\cos \theta\right|^{2}}\right. \\
& \left.+\frac{\left|\epsilon-\sin ^{2} \theta\right|+\sin ^{2} \theta}{\left|\sqrt{\epsilon-\sin ^{2} \theta}+\epsilon \cos \theta\right|^{2}}\right)\left|e^{-k \sqrt{\sin ^{2} \theta-\epsilon}\left|z_{0}\right|}\right|^{2},
\end{aligned}
$$

where $\Gamma_{0}$ is the emission probability of the excited atom placed in infinite vacuum, as given by Eq. (4) for $\epsilon=1$.

In Fig. 1, we show the behavior of the emission probability when the atom lies at the surface of a medium of real dielectric constant. The emission is enhanced if $\epsilon$ lies in the range between 0 and 1 , specially near the curve $\epsilon=\sin ^{2} \theta$, as shown in the figure. It should be noticed that within the region of the figure that lies on the right-hand side with respect to this curve the resulting emission field is made of evanescent waves inside the dielectric and propagating waves in vacuum. Therefore, if the atom is located deeper inside the dielectric, the emission tends to be suppressed within that region, whereas the rate remains unchanged on the left-hand side of the mentioned curve. Near the curve, the maximum values of the enhancement are reached for small values of $\epsilon$, giving rise to a very concentrated emission near the surface normal.

In Fig. 2, we have placed the atom in aluminum. We use tabulated optical data for the dielectric function of the metal. ${ }^{68}$ The dependence of the dielectric constant on the transition wavelength reduces the enhancement to a short range of frequencies, while the angular distribution shows that the emission is concentrated in a region of approximately $15^{\circ}$ around the surface normal. It should be stressed that the atom must be very close to the surface in order to achieve large enhancements. In particular, in Fig. 2(b) the distance from the atom to the surface is $20 \mathrm{~nm}$, and the maximum rate has decreased by an order of magnitude. 


\section{Far field in the medium containing the atom}

The calculation of the far field in the dielectric medium follows the same lines as above. Propagation at large distances will be possible only if $\epsilon$ is real and positive. Then, the total field is the interference of the direct emission from the atom and the field reflected at the surface.

In the far field limit, the total electric field can be expressed as $\mathbf{E}=\mathbf{f}^{\prime} e^{i k^{\prime} r} / r$, in analogy to the transmitted field considered above. Proceeding in the same way as in the preceding section, the probability of emission towards to dielectric is found to be

$$
\begin{aligned}
\frac{\Gamma(\epsilon, \theta)}{\Gamma_{0}}= & \frac{1}{2} \epsilon\left(2+\left|r_{s}\right|^{2}+\left|r_{p}\right|^{2}\right. \\
& \left.+2 \operatorname{Re}\left[\left(r_{s}-r_{p} \cos 2 \theta\right) e^{2 \mathrm{i} k^{\prime}\left|z_{0}\right|}\right]\right),
\end{aligned}
$$

where the reflection coefficients take the form

$$
\begin{aligned}
& r_{s}=\frac{\sqrt{\epsilon} \cos \theta-\sqrt{1-\epsilon \sin ^{2} \theta}}{\sqrt{\epsilon} \cos \theta+\sqrt{1-\epsilon \sin ^{2} \theta}}, \\
& r_{p}=\frac{\cos \theta-\sqrt{\epsilon} \sqrt{1-\epsilon \sin ^{2} \theta}}{\cos \theta+\sqrt{\epsilon} \sqrt{1-\epsilon \sin ^{2} \theta}},
\end{aligned}
$$

and the polar angle $\theta$ is referred to the negative $z$ axis (i.e., it lies within the $[0, \pi / 2]$ interval). In the case of $\epsilon=1$ there is no reflection, so that $r_{s}=r_{p}=0$ and we recover the familiar expression of Sec. II.

We compare in Fig. 3 the angle-integrated probability for emission towards the vacuum side versus the total emission rate for different values of $\epsilon$ and $z_{0}$. One can observe the interference between direct and reflected components of the total field as stated above, giving rise to oscillatory patterns with the atom-surface separation $\left|z_{0}\right|$. For large values of $\epsilon$, the transmitted field is very weak on both ways. On the contrary, small values of $\epsilon$ lead to large transmission near the surface, as already anticipated in Fig. 1.

\section{SPONTANEOUS EMISSION MODIFIED BY DIFFERENT GEOMETRIES}

So far we have studied the SE of an atom interacting with semi-infinite dielectric media. More complicated geometries will offer the possibility of collimating and producing strong enhancement or attenuation of the emission. In the following sections we will make a step further and focus on the behavior of the SE field in the presence of different nontrivial geometries.

The electromagnetic field created by the atom will be obtained in a similar way as in Sec. III. Let us start by Eq. (2) and its analogous for the magnetic field. Using the dipole approximation and Eq. (3), the direct electric and magnetic fields are found to be $\mathrm{b}^{13}$

$$
\begin{aligned}
\mathbf{E}^{d i r}(\mathbf{r})= & \frac{e^{\mathrm{i} k^{\prime} r}}{\epsilon r^{3}}\left[\left(k^{\prime 2} r^{2}+\mathrm{i} k^{\prime} r-1\right) \mathbf{d}-\left(k^{\prime 2} r^{2}+3 \mathrm{i} k^{\prime} r-3\right)\right. \\
& \times(\mathbf{d} \cdot \hat{\mathbf{r}}) \hat{\mathbf{r}}] \\
& \mathbf{H}^{d i r}(\mathbf{r})=-\mathrm{i} k \frac{e^{\mathrm{i} k^{\prime} r}}{r^{2}}\left(\mathrm{i} k^{\prime} r-1\right)(\hat{\mathbf{r}} \times \mathbf{d})
\end{aligned}
$$

where $\mathbf{r}$ is the spatial coordinate relative to the atom. Now, the total field will be obtained numerically by means of the $\mathrm{BEM}^{62}$ which permits solving Maxwell's equations exactly in the presence of arbitrarily shaped interfaces. The BEM consists in using interface charge and current distributions that are solved self-consistently by invoking the customary boundary conditions for the electromagnetic field. This is done at a discrete set of representative interface points. One ends up with a linear system of equations (the boundary conditions) and unknowns (the interface charges and currents). The dimensionality of the problem is reduced by focusing on the interfaces, and by taking care of the field propagation by means of homogeneous media Green functions. In practice, the problem becomes one dimensional in either axially symmetric geometries (for each azimuthal number $m$ ) or in interfaces with translational symmetry along a given direction. We will focus on axially symmetric nanostructures and the fields given by Eqs. (11) and (12) provide the inhomogeneous part of the mentioned system of linear equations. ${ }^{62}$ We will show calculations for cases in which the atomic electric dipole is oriented either parallel or perpendicular to the symmetry axis.

We will distinguish between direct and induced fields. The latter are the result of scattering of the direct field at the interfaces, so that one can write

$$
\begin{gathered}
\mathbf{E}(\mathbf{r})=\mathbf{E}^{d i r}(\mathbf{r})+\mathbf{E}^{i n d}(\mathbf{r}), \\
\mathbf{H}(\mathbf{r})=\mathbf{H}^{d i r}(\mathbf{r})+\mathbf{H}^{i n d}(\mathbf{r}) .
\end{gathered}
$$

with $\mathbf{E}^{\text {dir }}(\mathbf{r})$ and $\mathbf{H}^{\text {dir }}(\mathbf{r})$ given by Eqs. (11) and (12), respectively.

If the media under consideration are not absorbing (that is, their dielectric constants are all real) the emission rate $\Gamma$ and the atomic decay rate $\Gamma^{\text {decay }}$ take the same value. However, in the presence of absorption not all photons are emitted to the far field, and therefore $\Gamma<\Gamma^{\text {decay }}$. In any case, the total power emitted by the atom can be obtained by assuming that the induced electric and magnetic fields are approximately uniform inside an infinitesimally small region surrounding the atom (this assumption is reasonable when the atom is not located right on an interface, in which case one can always study the limit as the atom approaches the interface). The total emission probability can be calculated like in Eq. (4) by integrating the Poynting vector $\mathbf{S}$ derived from the BEM over a spherical surface fully contained in the same medium of dielectric function $\epsilon$ as the atom. One finds 


$$
\begin{aligned}
\hbar \Gamma^{\text {decay }}=\frac{2 c}{4 \pi \omega} \int d \Omega r^{2} \hat{\mathbf{r}} \cdot \operatorname{Re}\left[\mathbf{E}(\mathbf{r}) \times \mathbf{H}^{*}(\mathbf{r})\right]= & \frac{r^{2}}{2 \pi k} \int d \Omega \operatorname{Re}\left\{\frac{1}{\epsilon r^{3}}\left[-\frac{\mathrm{i} k}{r^{2}}\left(\mathrm{i} k^{\prime} r+1\right)\left\{\left(k^{\prime 2} r^{2}+\mathrm{i} k^{\prime} r-1\right)\left[\hat{\mathbf{r}} \cdot \mathbf{d} \times\left(\hat{\mathbf{r}} \times \mathbf{d}^{*}\right)\right]\right\}\right]\right\} \\
& +\frac{r^{2}}{2 \pi k} \int d \Omega \operatorname{Re}\left\{-\frac{\mathrm{i} k}{r^{2}}\left(\mathrm{i} k^{\prime} r+1\right) \hat{\mathbf{r}} \cdot\left[\mathbf{E}^{i n d}(\mathbf{r}) \times\left(\hat{\mathbf{r}} \times \mathbf{d}^{*}\right)\right] e^{-\mathrm{i} k^{\prime} r}\right\} \\
& +\frac{r^{2}}{2 \pi k} \int d \Omega \operatorname{Re}\left\{\frac{e^{\mathrm{i} k^{\prime} r}}{\epsilon r^{3}}\left(k^{\prime 2} r^{2}+\mathrm{i} k^{\prime} r-1\right) \hat{\mathbf{r}} \cdot\left[\mathbf{d} \times \mathbf{H}^{* i n d}(\mathbf{r})\right]\right\} .
\end{aligned}
$$

The term containing the induced electric and magnetic fields does not contribute when integrating over the solid angle $\Omega$ due to the hypothesis that they are uniform in the vicinity of the atom. Taking the limit $r \rightarrow 0$ and denoting $\mathbf{E}(\mathbf{r}=0)=\mathbf{E}_{0}$ we have

$$
\begin{aligned}
\hbar \Gamma^{\text {decay }}= & \frac{1}{2 \pi} \int d \Omega k^{3} \sqrt{\epsilon}\left[|\mathbf{d}|^{2}-|\mathbf{d} \cdot \hat{\mathbf{r}}|^{2}\right]+\frac{1}{2 \pi} \int d \Omega \\
& \times \operatorname{Re}\left\{-\mathrm{i}\left[\left(\mathbf{E}_{0}^{i n d} \cdot \mathbf{d}^{*}\right)-\left(\hat{\mathbf{r}} \cdot \mathbf{d}^{*}\right)\left(\hat{\mathbf{r}} \cdot \mathbf{E}_{0}^{i n d}\right)\right]\right\} \\
& +\lim _{r \rightarrow 0} \frac{1}{2 \pi k} \int d \Omega \operatorname{Re}\left\{\frac{-1}{\epsilon r} \hat{\mathbf{r}} \cdot\left[\mathbf{d} \times \mathbf{H}^{* i n d}(\mathbf{r})\right]\right\} .
\end{aligned}
$$

The first term is the same one that appears in Sec. II. The integration of the second term is straightforward as well. As for the third, we need to expand the magnetic field, $\mathbf{H}^{\text {ind }}(\mathbf{r})=\mathbf{H}_{0}^{\text {ind }}+(\mathbf{r} \cdot \vec{\nabla}) \mathbf{H}_{0}^{\text {ind }}$, since a uniform magnetic-field gives no contribution to the emission probability, and the $1 / r$ factor can still produce a finite contribution from the magnetic field gradient. After some algebra, and making use of Ampère-Maxwell's law, this third term becomes $(2 / 3) \operatorname{Im}\left(\mathbf{d}^{*} \cdot \mathbf{E}_{0}^{\text {ind }}\right)$, so that the total decay rate is found to $\mathrm{be}^{9,66}$

$$
\begin{aligned}
\Gamma^{\text {decay }} & =\frac{4}{3 \hbar} k^{3} \sqrt{\boldsymbol{\epsilon}}|\mathbf{d}|^{2}+\frac{4}{3 \hbar} \operatorname{Im}\left(\mathbf{d}^{*} \cdot \mathbf{E}_{0}^{\text {ind }}\right)+\frac{2}{3 \hbar} \operatorname{Im}\left(\mathbf{d}^{*} \cdot \mathbf{E}_{0}^{\text {ind }}\right) \\
& =\Gamma_{\epsilon}+\frac{2}{\hbar} \operatorname{Im}\left(\mathbf{d}^{*} \cdot \mathbf{E}_{0}^{\text {ind }}\right)
\end{aligned}
$$

Here, $\Gamma_{\epsilon}$ is the rate for the case where there is no induced field, that is, the dipole is isolated in a medium of dielectric constant $\epsilon$. This expression is very convenient and we have tested it by showing that the emission rate that it predicts agrees with the one obtained from the integral of the far-field Poynting vector when all materials under consideration have real dielectric function.

The emission probabilities presented in the sections below for arbitrary lossy materials are obtained by means of the far-field Poynting vector, which we have analyzed in angle of emission.

\section{FAR FIELD FOR TWO NANO-SPHERES}

The electromagnetic characteristics of the system formed by two spheres have been widely studied, for example the interaction between them and the hence induced multiple scattering ${ }^{70,71}$ the features of the system formed by two contacting spheres, ${ }^{72}$ the field-induced interaction force in two spheres, ${ }^{73}$ or the diffraction of electromagnetic waves. ${ }^{74}$ Nevertheless, we are not aware of any study of the behavior of the SE by an atom placed between two spheres which are very near from the other.

A big enhancement of the far-field emission can be achieved placing the electric dipole between two metallic spheres which are separated by a very small distance (a few $\mathrm{nm})$. In this case the atom can induce some dipolar surface charges on the spheres, giving rise to a strong modification of the field. To illustrate the fact that for absorbent media $\Gamma<\Gamma^{\text {decay }}$ (i.e., part of the decay is converted into emission and the rest is absorbed by the material), we show in Fig. 4 both quantities for the two-sphere system. $\Gamma^{\text {decay }}$ has been calculated from Eq. (13), while for $\Gamma$ we have used instead the far-field Poynting vector. In Figs. 5(a) and 5(b) it is shown this behavior when the dipole is placed between two gold spheres of $50 \mathrm{~nm}$ of radius. There are some wavelengths, which coincide with the plasmon resonance of the material, for which the enhancement is dramatic, but only for the case where the dipole orientation is parallel to the imagi-

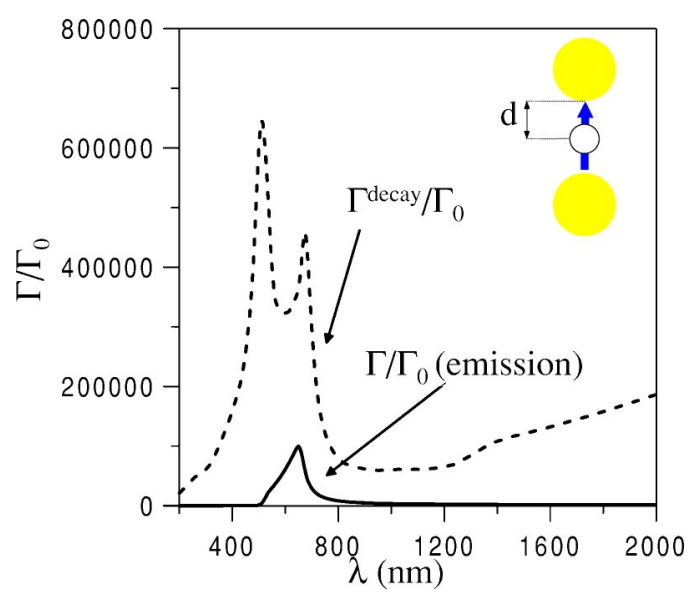

FIG. 4. (Color online) Decay and far-field emission probabilities, normalized to vacuum, for an atom placed at a distance $d$ $=1 \mathrm{~nm}$ from the surface of two gold spheres of $50 \mathrm{~nm}$ of radius and situated between them (see text for more details). 

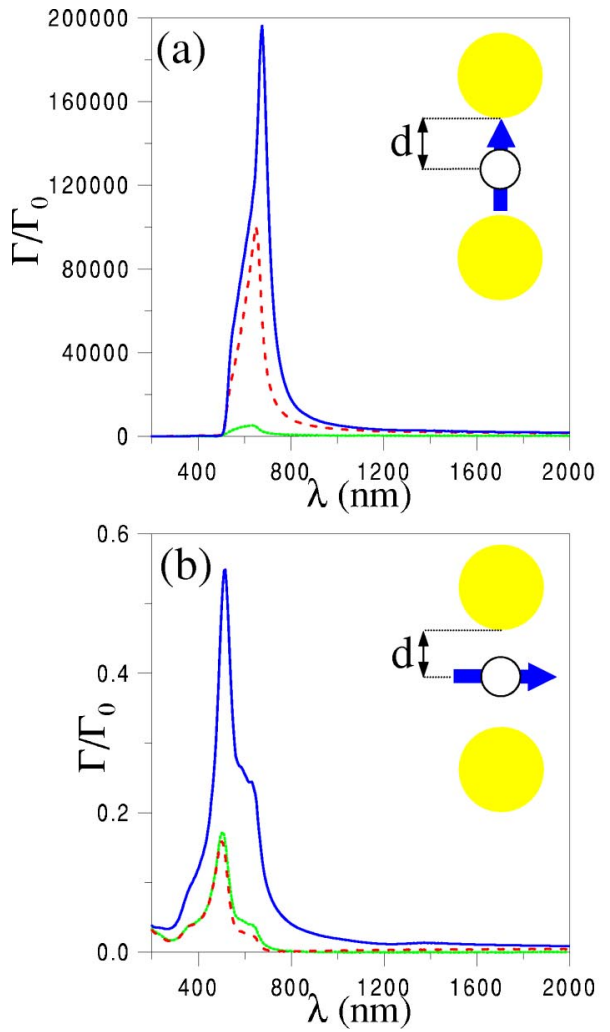
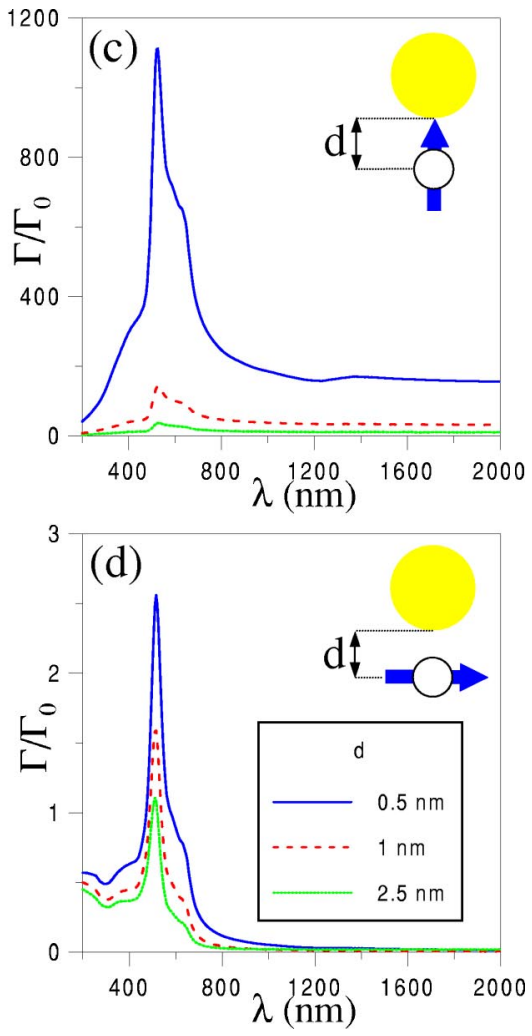

FIG. 5. (Color online) (a) Spontaneous emission rate normalized to vacuum for an atom situated between two gold spheres of radius $50 \mathrm{~nm}$, and with its electric dipole oriented parallel to the line that crosses the center of the spheres. Three different sphere separations are considered, so that the distance from the atom to each of the spheres is $0.5,1$, or $2.5 \mathrm{~nm}$ (the rate increases with decreasing distance). (b) Same as (a) when the dipole is oriented perpendicular with respect to the one in (a). (c) (d) Same as (a) and (b), respectively, but with only one sphere. nary line which goes from one sphere to the other. For the orientation perpendicular to this one it is given suppression of the emission. This difference is due to the characteristics of the induced dipoles in the spheres; in the former case they add its contribution, while in the latter they cancel it.

It can also be seen that the enhancement is highly dependent of the distance between spheres, growing dramatically with it. Also the position of the maximum varies, although slightly, with this parameter; it decreases when increasing the gap between spheres, until reaching a constant value of about $480 \mathrm{~nm}$ which corresponds to the case of only one sphere [Fig. 5(c)]. Note that in this last case this position does not change when placing the atom at different distances of the sphere.

\section{FAR FIELD FOR GOLD NANORINGS}

Next, we will apply our formalism to gold rings of several heights, as shown in the inset of Fig. 6. Recently it has been shown ${ }^{69}$ that this kind of rings show infrared plasmon resonances in the extinction cross-section spectrum which are due only to its shape, as they do not appear in solid disks. In our study, the atom will be placed at several distances of the center of the ring. We calculate the probability emission related to the case in which there is only vacuum surrounding the atom from the far field given by the boundary element method. Results are shown in Fig. 6, using tabulated optical data for the dielectric function of $\mathrm{Au} .^{68}$

A strong enhancement of the emission is observed for some energies when the dipole is oriented perpendicular to the ring axis. This enhancement is maximum when the atom is in the center of the ring, decreasing with the height, but being still considerable high even when the atom is above the ring. The wavelengths, at which the peaks occur, grow with the height of the ring. On the other way, when the dipole orientation is parallel to the ring axis, then a suppression is observed, for no probability bigger than the one for the vacuum is given.

Concerning the directionality of the emission, in general it has not been observed a strong collimation of the resulting field, so this kind of systems can be used in order to improve the emission, but not for concentrate it within small angles.

As it happened with the spheres, the directionality of the radiation emitted for this system is approximately the same as for the single dipole without any interaction.

It should be noticed that for rings the effect is exactly the opposite as for the spheres: suppression for dipole orientation along the revolution symmetry axis of the system and enhancement for perpendicular orientation. So, with either one of these two systems we can amplify an arbitrary dipolar emission, as will be seen in the following section.

\section{FAR FIELD FOR GRATINGS}

So far, we have studied systems in which the enhancement of the SE can be very high. Nevertheless, the other main goal of this work, the collimation of the resulting field, has not been achieved neither for rings nor for spheres, although semi-infinite media already provided a way of doing this.

Recently, ${ }^{33,54,55}$ grating geometries have been applied to collimate different kinds of optical emissions. In this work we will construct a very simple axially symmetric grating formed by a certain number of grooves of cosine shape (see 


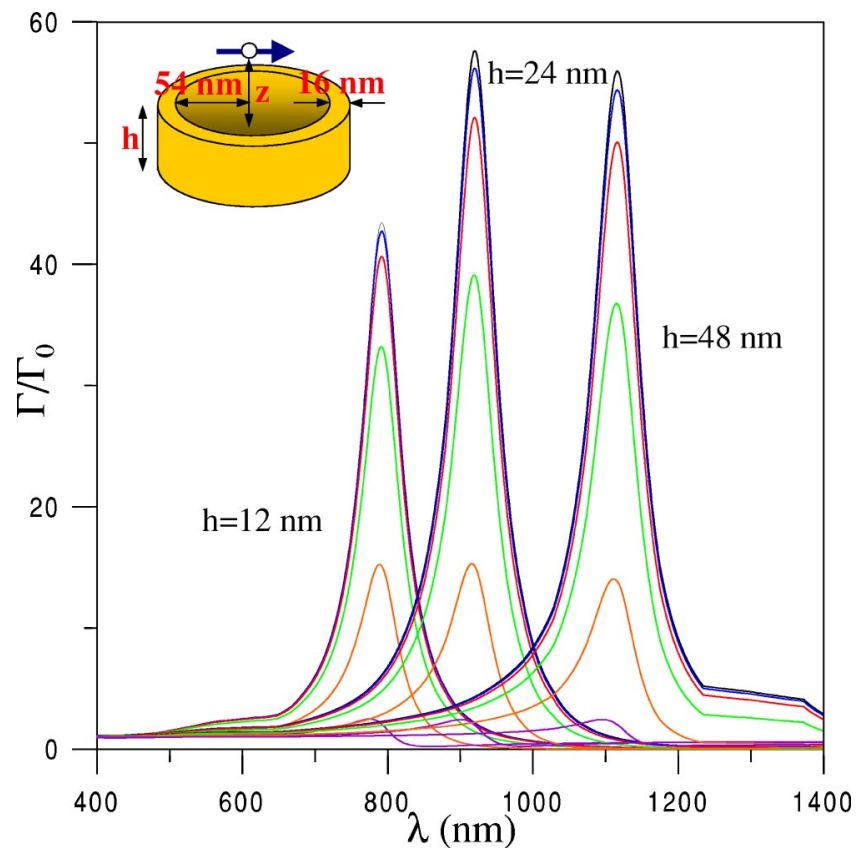

FIG. 6. (Color online) Emission probability normalized to vacuum from an atom placed at the axis of a nano-ring of height $h$ at a distance $z$ from its center. The transition electric dipole is oriented perpendicular to the ring axis. The three peaks correspond to different values of $h$ (see labels). Within each peak, the values of $z$ are (from the top to the bottom) $0,6,12,24,48$, and $96 \mathrm{~nm}$, respectively.

inset in Fig. 7), which will give big directionality of the emitted field. We will change the number of grooves, while maintaining fixed the groove period $s$, the groove height $h$, and the thickness of the film $b$. We use the dielectric constant for silver at $\lambda=600 \mathrm{~nm}(\epsilon=-16+0.5 \mathrm{i}) .{ }^{68}$ All the geometrical parameters will be expressed in terms of this wavelength. In this way, the results obtained here will be a consequence mostly of the geometry when the grating material is made of a good metal, even if not perfect. Indeed, for other metalliclike values of the dielectric constant (for example, $\epsilon=-25$ $+\mathrm{i}$, which corresponds to silver and copper near $800 \mathrm{~nm}$ ), the directionality dependence with $s / \lambda$ is qualitatively the same and quantitatively very similar (the results of Fig. 7 are almost the same when this new value of $\epsilon$ is used); hence, one can conclude that the results can be extrapolated to the desired emission frequency just by changing the dimensions of the grating to the corresponding range of wavelengths (visible, infrared). In particular, a similar type of grating geometry is well known in the microwave antenna community. ${ }^{51,52}$

In Fig. 7 we show the ratio between the emission probability for this system and the emission probability in vacuum, in terms of the ratio between the size of the grating and the emission wavelength, and the polar angle $\theta$, in the case in which there are 2, 5, and 10 grooves. Two possible dipole orientations (parallel and perpendicular to the grating axial symmetry axis) are shown. For small distances between the atom and the grating (less than $s / 20$ ) the results are practically equivalent. We show here the results when this distance is $s / 25$.

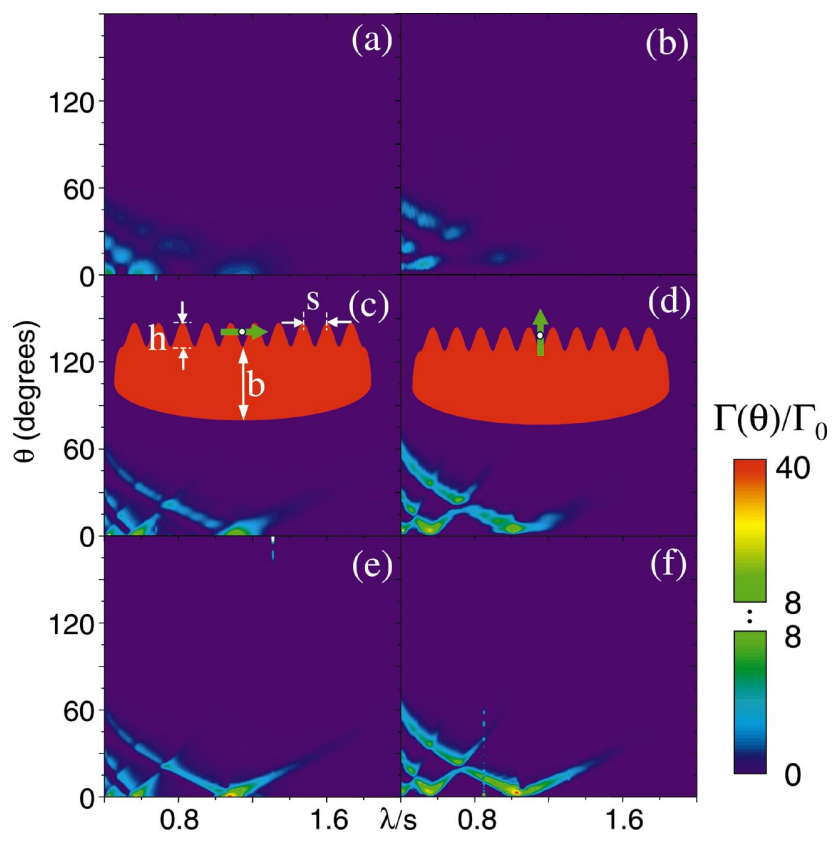

FIG. 7. (Color) (a) Angle-resolved emission probability for an atom located in the center of a silver grating consisting of two axially symmetric grooves. The intensity has been integrated over azimuthal directions and normalized to the total emission probability in the absence of the grating, $\Gamma_{0}$. The transition dipole moment $\mathbf{d}$ is taken perpendicular to the grating axis. The grating has cosinelike profile with parameters $h=0.2 s$ and $b=0.6 s$, where $h$ is the groove depth, $s$ is the groove pitch, and $b$ is the thickness of the film (see inset, not to scale). The atom is located at a distance $0.04 \mathrm{~s}$ above the silver surface. The emission wavelength is $\lambda=600 \mathrm{~nm}$. (b) Same as (a), for $\mathbf{d}$ parallel to the axis of symmetry. (c)-(d) Same as (a) and (b), respectively, but for five grooves (this is the geometry actually plotted in the inset). (e)-(f) Same as (a) and (b), respectively, but for 10 grooves. (b), (d), and (f) should be multiplied by a factor 3 in order to get the actual emission probability.

The first effect that is clearly seen is that there is a strong collimation for a very defined set of frequencies for both orientations of the dipole. When the dipole is parallel to the axis, the maximum wavelength increases with the number of grooves, while for the case in which the dipole is perpendicular to the axis the maximum is nearly independent of the number of grooves, being situated around $\lambda / s=1.1$. For the first case, as the emission is suppressed at $\theta=0$, the angle at which the emission peak occurs decreases as well with increasing the number of grooves; for a dipole perpendicular to the axis, this maximum occurs always at $\theta=0$. Interestingly, the emission is concentrated within a very small range of polar angles, and this range is decreasing dramatically as the number of grooves is bigger.

The dependence of the results on the geometrical parameters of the grating is shown in Fig. 8 for the case in which the grating consists of five grooves. We choose to make the comparisons in the case of a perpendicular dipole to the symmetry axis, because the value of $\lambda / s$ at which the maximum emission is achieved does not depend appreciably on the number of grooves. Maintaining the period of the groove $s$ fixed, it can be seen that the role of the groove height $h$ is 


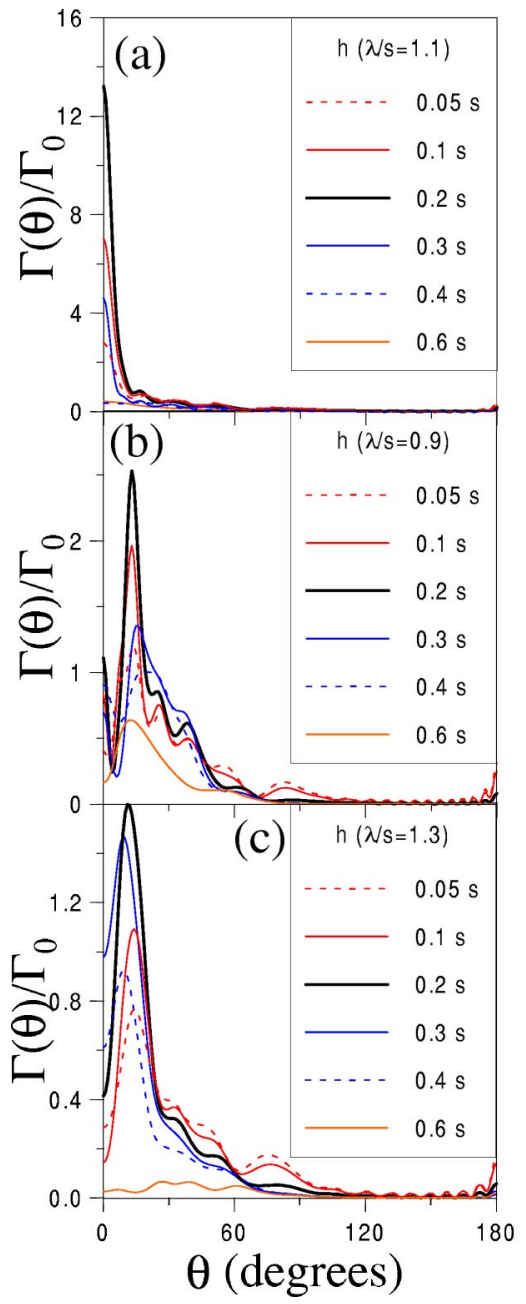

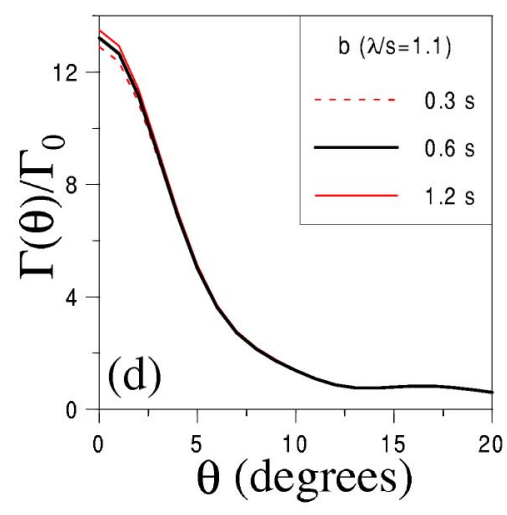
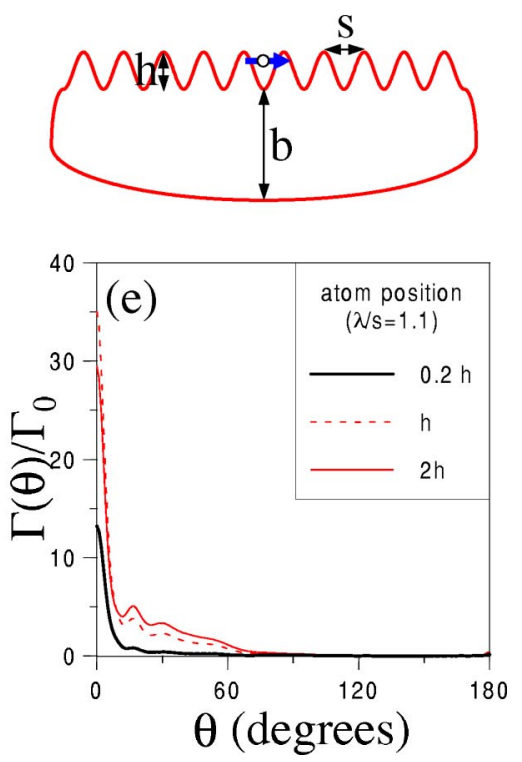

FIG. 8. (Color online) Dependence of the emission probability on the geometric parameters for an atom placed in the center of an axially symmetric grating consisting of five grooves (see inset). The dipole is oriented perpendicularly to the symmetry axis. (a)-(c) Dependence with the height of the grooves within the main resonance $[\lambda / s=1.1$, see Fig. $7(\mathrm{c})]$ and outside it $(\lambda / s$ $=0.9$ and 1.3), respectively. (d) Dependence with the depth of the lower part of the grating at resonance. (e) Dependence with the distance between the atom and the grating, under resonance conditions. The parameters that are not changed take the same values as in Fig. $7(h=0.2 s, b=0.6 s$, and atom height equal to $0.04 \mathrm{~s}$ ).
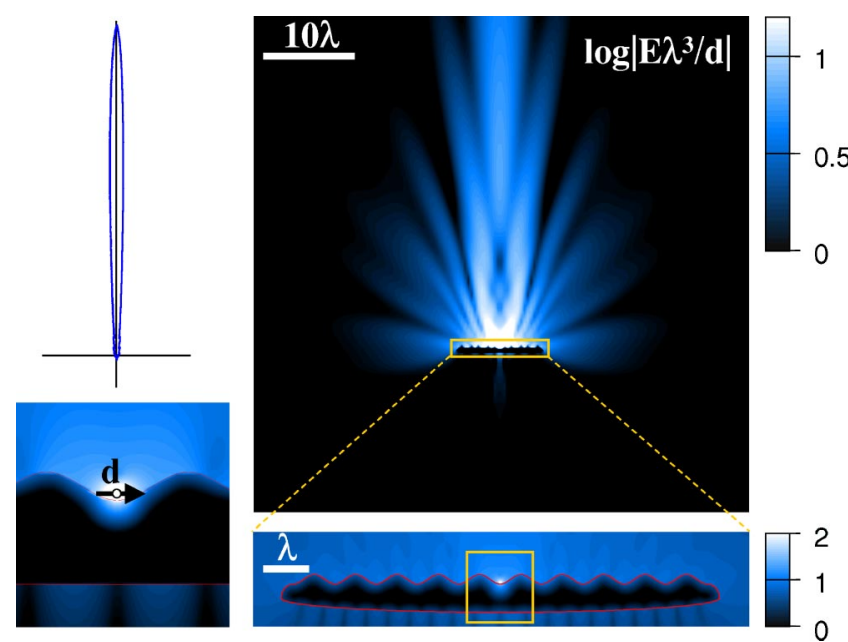

FIG. 9. (Color online) Logarithm of the electric field strength near a five-groove silver grating under the same conditions as in Fig. 7(c) (transition dipole perpendicular to axis of symmetry) for a wavelength $\lambda=600 \mathrm{~nm}$. The angular distribution of the emission intensity is shown in the upper left corner in linear scale.
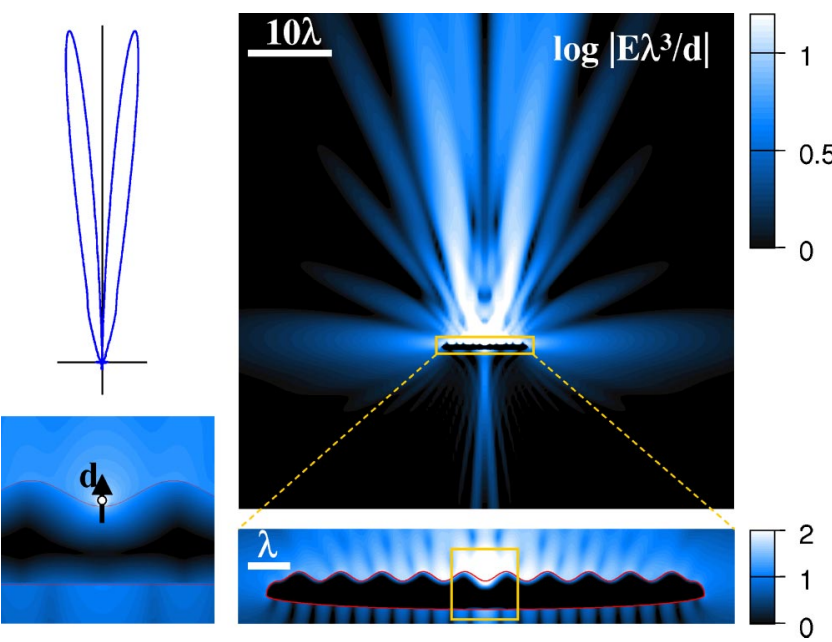

FIG. 10. (Color online) Logarithm of the electric field strength near a five-groove silver grating under the same conditions as in Fig. 7(d) (transition dipole parallel to axis of symmetry) for a wavelength $\lambda=600 \mathrm{~nm}$. The angular distribution of the corresponding emission intensity is shown in the upper left corner in linear scale. 

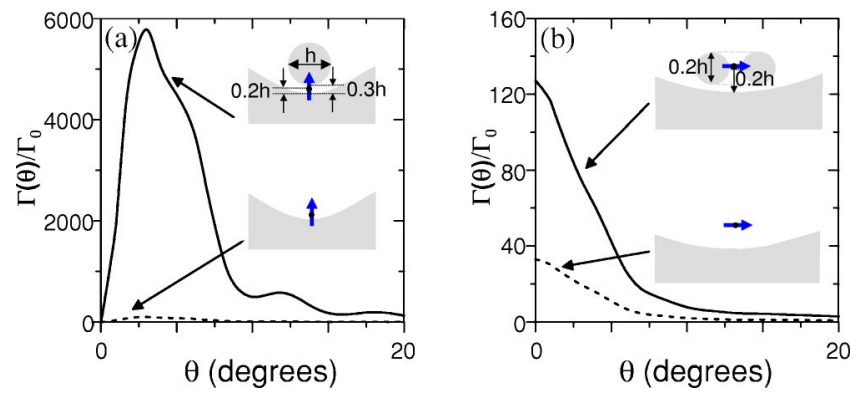

FIG. 11. (Color online) (a) Polar-angle dependence of the spontaneous emission probability under the resonance conditions of Fig. $7(\mathrm{f})(\lambda / s=1.01)$, adding a silver sphere to the system in which the dipole is along the symmetry axis of the grating, which is formed by 10 grooves. (b) Same as (a), but adding a silver torus to the system in which the dipole is oriented perpendicular to the symmetry axis of the grating. The resonance conditions of Fig. 7(e) $(\lambda / s$ $=1.1$ ) are fulfilled.

crucial, as the angle resolution can be strongly modified with a slight variation of its value. With $h=0.2 s$ we get both a maximum of the emission and a minimum in the peak width [Fig. 8 (a)]. This value of $h$ also seems to be the most accurate for values of $\lambda / s$ situated outside the resonance [Figs. 8(b) and 8(c)]. On the contrary, the thickness of the film $b$ is almost irrelevant when studying the upwards emission (small values of $\theta$ ), as can be seen in Fig. 8(d). Finally, placing the atom at a higher distance from the grooves maximizes the total emission, while losing directionality, as the obtained peaks are wider [Fig. 8(e)].

The properties of the field in the vicinity of the grating are shown in Figs. 9 and 10, for two possible orientations of the atomic electric dipole, for a grating composed of five grooves, and for the values of $\lambda / s$ which provide the maximum far-field emission. In both figures, the largest plot shows the relevant distance at which focusing begins, while the small ones show the regions closer to the emitter. A straightforward comparison can be made between the largest plot and the angular far-field pattern, shown in the upper left part of the figures.

Although the enhancement itself is not very important (the maximum for ten gratings is a factor 160 for parallel dipole and 50 for perpendicular), one can combine this geometry with the ones discussed in preceding sections, so that the main goal of strong emission enhancement and focused emission can be obtained simultaneously. Therefore, in Fig. 11 we show the results of the far-field emission under resonance conditions for a ten-groove grating when adding to the latter a silver sphere in the case of the dipole parallel to the axis, and a small silver torus in the case of the dipole perpendicular to the axis. The emission is enhanced by a factor of 60 in the first case, while the enhancement is smaller in the second one, mainly because of the reduced dimensions of the torus that can fit into the system.

\section{CONCLUSIONS}

The modification of the light emission from excited atoms by means of the interaction of the SE field with the environ- ment has been studied in the presence of several complex geometries that lead to global enhancement of the emission rate or marked directionality of the emission field.

If the atom is placed at the planar surface of a material, the emission is focused along directions of emission relative to the surface normal that depend on the material dielectric function $\epsilon,{ }^{31,33}$ and in particular, the emission is directed along the surface normal for vanishing $\epsilon$ (Fig. 1). For absorbing materials such as aluminum, this strong directionality takes place only at wavelengths near the bulk plasmon resonance (Fig. 2), and therefore such a simple device could be used as a filter or as a lens, in clear analogy to the nearfield lenses recently proposed by Pendry. ${ }^{75}$

Nanostructured, finite objects can also be used to modify the SE in the same fashion. With the help of the boundary element method for arbitrary shape geometries, ${ }^{62}$ we have calculated the SE rate from an excited atom situated near structures of this kind for different orientations of the associated atomic electric dipole. Remarkable global enhancement of the emission has been found near plasmon resonance frequencies in gold spheres (Fig. 5) and nano-rings (Fig. 6). Also, strong directionality of the emission has been found when the atom is located near a grating. ${ }^{33,51,52}$ Axially symmetric gratings made of real silver metal have been explored. In this case, the SE field can be collimated around the grating axis within an angular window of $5^{\circ}$ (Figs. 9 and 10). Combining some of the above geometries (e.g., gratings and spheres or rings) both effects of directionality and enhancement are present simultaneously in the resulting SE far field (Fig. 11).

The present work provides new means for obtaining emission antennas by exploring the directionality phenomena discussed above. We also hope that it can stimulate the use of nanostructures to modify the electromagnetic field in the near-field domain, and as a consequence, in the far-field produced by localized sources, not only in the case of SE.

\section{ACKNOWLEDGMENTS}

This work has been supported in part by the Basque Departamento de Educación, Universidades e Investigación, the University of the Basque Country UPV/EHU (Contract No. 00206.215-13639/2001) and the Spanish Ministerio de Ciencia y Tecnología (Contract No. MAT2001-0946).

\section{APPENDIX: INTEGRATION OF THE FAR-FIELD COMPONENTS IN PLANAR INTERFACES}

The integration of the field given by Eqs. (7) and (6) is carried out in this appendix to yield Eq. (8).

Expanding Eq. (7), the transmitted electric field in vacuum takes the form

$$
\mathbf{E}(\mathbf{r})=\int d \mathbf{Q E}(\mathbf{Q}) e^{\mathrm{i} \mathbf{Q} \cdot \mathbf{R}-\Delta z-\Delta^{\prime}\left|z_{0}\right|},
$$

where the Cartesian components of $\mathbf{E}(\mathbf{Q})$ are given by 
TABLE I. Values of $I=\int d \varphi_{Q} P\left(Q_{x}, Q_{y}\right) e^{\mathrm{iQ} \cdot \mathbf{R}}$ for different polynomials $P\left(Q_{x}, Q_{y}\right)$. We denote $J_{m} \equiv J_{m}(Q R)$.

\begin{tabular}{lc}
\hline \hline$P\left(Q_{x}, Q_{y}\right)$ & $I$ \\
\hline 1 & $2 \pi J_{0}$ \\
$Q_{x}$ & $2 \mathrm{i} \pi Q \cos \varphi J_{1}$ \\
$Q_{y}$ & $2 \mathrm{i} \pi Q \sin \varphi J_{1}$ \\
$Q_{x}^{2}$ & $\pi Q^{2}\left(\cos ^{2} \varphi\left(-J_{2}+J_{0}\right)+\sin ^{2} \varphi\left(J_{2}+J_{0}\right)\right)$ \\
$Q_{x} Q_{y}$ & $-\pi Q^{2} \cos \varphi \sin J_{2}$ \\
$Q_{y}^{2}$ & $\pi Q^{2}\left(\cos ^{2} \varphi\left(J_{2}+J_{0}\right)+\sin ^{2} \varphi\left(-J_{2}+J_{0}\right)\right)$ \\
\hline \hline
\end{tabular}

$$
\begin{aligned}
E_{x}(\mathbf{Q})= & \frac{t_{s}}{2 \pi \Delta^{\prime}} \frac{k^{2}}{Q^{2}}\left(d_{x} Q_{y}^{2}-d_{y} Q_{x} Q_{y}\right) \\
& -\frac{t_{p}}{2 \pi} \frac{\Delta}{\Delta^{\prime}} \frac{1}{Q^{2} \sqrt{\epsilon}}\left(\Delta^{\prime} d_{x} Q_{x}^{2}+\Delta^{\prime} d_{y} Q_{x} Q_{y}\right. \\
& \left.+\mathrm{i} Q^{2} d_{z} Q_{x}\right) \\
E_{y}(\mathbf{Q})= & \frac{t_{s}}{2 \pi \Delta^{\prime}} \frac{k^{2}}{Q^{2}}\left(-d_{x} Q_{x} Q_{y}+d_{y} Q_{x}^{2}\right) \\
& -\frac{t_{p}}{2 \pi} \frac{\Delta}{\Delta^{\prime}} \frac{1}{Q^{2} \sqrt{\epsilon}}\left(\Delta^{\prime} d_{x} Q_{x} Q_{y}+\Delta^{\prime} d_{y} Q_{y}^{2}\right. \\
& \left.+\mathrm{i} Q^{2} d_{z} Q_{y}\right), \\
E_{z}(\mathbf{Q})=- & \frac{t_{p}}{2 \pi \Delta^{\prime} \sqrt{\epsilon}}\left(\mathrm{i} \Delta^{\prime} d_{x} Q_{x}+\mathrm{i} \Delta^{\prime} d_{y} Q_{y}-Q^{2} d_{z}\right)
\end{aligned}
$$

$\Delta=\sqrt{Q^{2}-k^{2}-i 0^{+}}$, and $t_{p}$ and $t_{s}$ are Fresnel's plane-wave transmission coefficients. ${ }^{13}$

The integration of Eq. (A1) can be made in two steps. The first one is the integration over the angular coordinate of $\mathbf{Q}$, which gives rise to Bessel functions ${ }^{76}$ according to the rule provided in Table I.
The remaining integral in $\mathrm{Q}$ can be done analytically in the $r \rightarrow \infty$ limit. The Bessel functions can be approximated in this limit as ${ }^{76}$

$$
J_{m}(Q R) \approx \frac{1}{2 \sqrt{2 \pi Q R}}\left(e^{\mathrm{i}(Q R-\pi / 4-m \pi / 2)}+e^{-\mathrm{i}(Q R-\pi / 4-m \pi / 2)}\right)
$$

Here, the emission angle enters via $R=r \sin \theta, \quad z$ $=r \cos \theta$. The positive exponential in Eq. (A2) takes the form

$$
e^{\mathrm{i} Q R} e^{-\Delta z}=e^{\mathrm{i} f(Q) r},
$$

where

$$
\begin{aligned}
f(Q) & =Q \sin \theta+\sqrt{k^{2}-Q^{2}} \cos \theta \\
& =k+\alpha\left(Q-Q_{0}\right)^{2}+O\left(Q-Q_{0}\right)^{3}
\end{aligned}
$$

$\alpha=-1 /\left(2 k \cos ^{2} \theta\right)$, and $Q_{0}=k \sin \theta$ is such that $f^{\prime}\left(Q_{0}\right)=0$ $\left(\right.$ stationary phase approximation $\left.^{77}\right)$. The negative exponential does not lead to a stationary phase, and therefore, it can be disregarded.

The integral over $r$ can then be approximated as

$$
\int_{0}^{Q_{\max }} d Q F(Q) e^{\mathrm{i} f(Q) r} \approx F\left(Q_{0}\right) e^{\mathrm{i} k r} \int_{-Q_{0}}^{Q_{\max }-Q_{0}} d Q Q^{\prime} e^{\mathrm{i} \alpha Q^{\prime 2} r},
$$

which in the $r \rightarrow \infty$ limit leads to

$$
\int_{0}^{Q_{\max }} d Q F(Q) e^{\mathrm{i} f(Q) r} \stackrel{r \rightarrow \infty}{\rightarrow} F\left(Q_{0}\right) e^{\mathrm{i} k r}(1-\mathrm{i}) \sqrt{\frac{\pi k}{r}} \cos \theta .
$$

Substituting $Q$ by $Q_{0}=k \sin \theta$ one readily obtains Eq. (8). The results of Sec. III C (direct and reflected part) are obtained by using $Q_{0}=k \sqrt{\epsilon} \sin \theta$.
${ }^{1}$ E.M. Purcell, Phys. Rev. 69, 681 (1946).

${ }^{2}$ G. Björk, S. Machida, Y. Yamamoto, and K. Igeta, Phys. Rev. A 44, 669 (1991).

${ }^{3}$ E. Yablonovitch, Phys. Rev. Lett. 58, 2059 (1987).

${ }^{4}$ S. John and T. Quang, Phys. Rev. A 50, 1764 (1994).

${ }^{5}$ S. Fan, P.R. Villeneuve, J.D. Joannopoulos, and E.F. Schubert, Phys. Rev. Lett. 78, 3294 (1997).

${ }^{6}$ S. Enoch, B. Gralak, and G. Tayeb, Appl. Phys. Lett. 81, 1588 (2002); S. Enoch, G. Tayeb, P. Sabouroux, N. Guérin, and P. Vincent, Phys. Rev. Lett. 89, 213902 (2002).

${ }^{7}$ E.A. Hinds, Adv. At., Mol., Opt. Phys. 28, 237 (1991).

${ }^{8}$ D. Kleppner, Phys. Rev. Lett. 47, 233 (1981).

${ }^{9}$ V.V. Klimov, Opt. Commun. 211, 183 (2002).

${ }^{10}$ V.V. Klimov, M. Ducloy, and V.S. Letokhov, Eur. Phys. J. D 20, 133 (2002).

${ }^{11}$ F.L. Kien, N.H. Quang, and K. Hakuta, Opt. Commun. 178, 151
(2000).

${ }^{12}$ T. Brage, P.G. Judge, and C.R. Proffitt, Phys. Rev. Lett. 89, 281101 (2002).

${ }^{13}$ J. D. Jackson, Classical Electrodynamics (J Wiley, New York, 1962).

${ }^{14}$ P. Goy, J.M. Raimond, M. Gross, and S. Haroche, Phys. Rev. Lett. 50, 1903 (1983).

${ }^{15}$ W. Jhe, A. Anderson, E.A. Hinds, D. Meschede, L. Moi, and S. Haroche, Phys. Rev. Lett. 58, 666 (1987).

${ }^{16}$ H.-B. Lin, J.D. Eversole, C.D. Merritt, and A.J. Campillo, Phys. Rev. A 45, 6756 (1992).

${ }^{17}$ H.P. Urbach and G.L.J.A. Rikken, Phys. Rev. A 57, 3913 (1998).

${ }^{18}$ G.L.J.A. Rikken, Physica B 204, 353 (1995).

${ }^{19}$ H.T. Dung, L. Knoll, and D.-G. Welsch, Recent Res. Dev. Opt. 1, 225 (2001).

${ }^{20}$ E.P. Petrov, V.N. Bogomolov, I.I. Kalosha, and S.V. Gaponenko, 
Phys. Rev. Lett. 81, 77 (1998).

${ }^{21}$ X.-H. Wang, R. Wang, B.-Y. Gu, and G.-Z. Yang, Phys. Rev. Lett. 88, 093902 (2002).

${ }^{22}$ Y. Yang and S.Y. Zhu, Phys. Rev. A 61, 043809 (2000).

${ }^{23}$ S.G. Romanov, T. Maka, C.M. Sotomayor Torres, M. Muller, and R. Zentel, J. Appl. Phys. 91, 9426 (2002).

${ }^{24}$ I. Bulu, H. Caglayan, and E. Ozbay, Phys. Rev. B 67, 205103 (2003).

${ }^{25}$ S.Y. Lin, J. Moreno, and J.G. Fleming, Appl. Phys. Lett. 83, 380 (2003)

${ }^{26}$ J.M. Gérard, B. Sermage, B. Gayral, B. Legrand, E. Costard, and V. Thierry-Mieg, Phys. Rev. Lett. 81, 1110 (1998).

${ }^{27}$ J.J. Sanchez-Mondragon, N.B. Narozhny, and J.H. Eberly, Phys. Rev. Lett. 51, 550 (1983).

${ }^{28}$ K. Tanaka, T. Nakamura, W. Takamatsu, M. Yamanishi, Y. Lee, and T. Ishihara, Phys. Rev. Lett. 74, 3380 (1985).

${ }^{29}$ A. Kamli and M. Babiker, Phys. Rev. A 62, 043804 (2000).

${ }^{30}$ S. Sachdev, Phys. Rev. A 29, 2627 (1984).

${ }^{31}$ R.M. Amos and W.L. Barnes, Phys. Rev. B 55, 7249 (1997).

${ }^{32}$ J. Eschner, C. Raab, F. Schmidt-Kaler, and R. Blatt, Nature (London) 413, 495 (2001).

${ }^{33}$ P. Andrew and W.L. Barnes, Phys. Rev. B 64, 125405 (2001).

${ }^{34}$ A. Bouhelier, M. Beversluis, A. Hartschuh, and L. Novotny, Phys. Rev. Lett. 90, 013903 (2003).

${ }^{35}$ R.G. Hulet, E.S. Hilfer, and D. Kleppner, Phys. Rev. Lett. 55, 2137 (1985).

${ }^{36}$ T. Gutbrod, M. Bayer, A. Forchel, P.A. Knipp, T.L. Reinecke, A. Tartakovskii, V.D. Kulakovskii, N.A. Gippius, and S.G. Tikhodeev, Phys. Rev. B 59, 2223 (1999).

${ }^{37}$ M. Bayer, T.L. Reinecke, F. Weidner, A. Larionov, A. McDonald, and A. Forchel, Phys. Rev. Lett. 86, 3168 (2001).

${ }^{38}$ A. Görlitz, A.P. Chikkatur, and W. Ketterle, Phys. Rev. A 63, 041601 (2001).

${ }^{39}$ H. Chew, J. Chem. Phys. 87, 1355 (1987).

${ }^{40}$ V.V. Klimov, M. Ducloy, and V.S. Letokhov, Quantum Electron. 31, 569 (2001).

${ }^{41}$ V.V. Klimov, M. Ducloy, and V.S. Letokhov, J. Mod. Opt. 44, 1081 (1997); Phys. Rev. A 59, 2996 (1999).

${ }^{42}$ R.E. Benner, P.W. Barber, J.F. Owen, and R.K. Chang, Phys. Rev. Lett. 44, 475 (1980).

${ }^{43}$ S.C. Ching, H.M. Lai, and K. Young, J. Opt. Soc. Am. B 4, 2004 (1987).

${ }^{44}$ J. Parker and C.R. Stroud, Jr., Phys. Rev. A 35, 4226 (1987).

${ }^{45}$ A.J. Campillo, J.D. Eversole, and H.-B. Lin, Phys. Rev. Lett. 67, 437 (1991).

${ }^{46}$ H. Xu, E.J. Bjerneld, M. Käll, and L. Börjesson, Phys. Rev. Lett. 83, 4357 (1999).

${ }^{47}$ V.A. Shubin, W. Kim, V.P. Safonov, A.K. Sarychev, R.L. Armstrong, and V.M. Shalaev, J. Lightwave Technol. 17, 2183 (1999).

${ }^{48}$ H. Xu, J. Aizpurua, M. Käll, and P. Apell, Phys. Rev. E 62, 4318 (2000).

${ }^{49}$ J.P. Kottmann, O.J.F. Martin, D.R. Smith, and S. Schultz, Chem. Phys. Lett. 341, 1 (2001).
${ }^{50}$ H. Xu and M. Käll, Phys. Rev. Lett. 89, 246802 (2002).

${ }^{51}$ G.L. James, Electron. Lett. 13, 293 (1977).

${ }^{52}$ L. Shafai and A.A. Kishk, in Microwave Horn and Feeds, edited by A.D. Olver, P.J.B. Clarricoats, A.A. Kishk, and L. Shafa, IEE Electromagnetic Waves Series 39 (IEEE Press, New York, 1994).

${ }^{53}$ T.W. Ebbesen, H.J. Lezec, H.F. Ghaemi, T. Thio, and P.A. Wolff, Nature (London) 391, 667 (1998).

${ }^{54}$ H.J. Lezec, A. Degiron, E. Devaux, R.A. Linke, L. MartínMoreno, F.J. García-Vidal, and T.W. Ebbesen, Science 297, 820 (2002).

${ }^{55}$ L. Martín-Moreno, F.J. García-Vidal, H.J. Lezec, A. Degiron, and T.W. Ebbesen, Phys. Rev. Lett. 90, 167401 (2003).

${ }^{56}$ F. Yang and J.R. Sambles, Phys. Rev. Lett. 89, 063901 (2002).

${ }^{57}$ F.J. García-Vidal, H.J. Lezec, T.W. Ebbesen, and L. MartínMoreno, Phys. Rev. Lett. 90, 213901 (2003).

${ }^{58}$ S.A. Tretyakov and C.R. Simovski, Microwave Opt. Technol. Lett. 27, 46 (2000).

${ }^{59}$ O.J.F. Martin, C. Girard, and A. Dereux, Phys. Rev. Lett. 74, 526 (1995).

${ }^{60}$ O.J.F. Martin, C. Girard, D.R. Smith, and S. Schultz, Phys. Rev. Lett. 82, 315 (1999).

${ }^{61}$ M. Paulus, P. Gay-Balmaz, and O.J.F. Martin, Phys. Rev. E 62, 5797 (2000).

${ }^{62}$ F.J. García de Abajo and A. Howie, Phys. Rev. Lett. 80, 5180 (1988); Phys. Rev. B 65, 115418 (2002).

${ }^{63}$ N. F. Mott and I. N. Sneddon, Wave Mechanics and Its Applications (Oxford University Press, London, 1948).

${ }^{64}$ F.J. García de Abajo, C.S. Fadley, and M.A. Van Hove, Phys. Rev. Lett. 82, 4126 (1999).

${ }^{65}$ V.V. Kocharovsky, V1.V. Kocharovsky, and A.A. Belyanin, Phys. Rev. Lett. 76, 3285 (1996).

${ }^{66}$ R.R. Chance, A. Prock, and R. Sylbey, Adv. Chem. Phys. 37, 1 (1978).

${ }^{67}$ F.J. García de Abajo and L.A. Blanco, Phys. Rev. B 67, 125108 (2003).

${ }^{68}$ E. D. Palik, Handbook of Optical Constants of Solids (Academic Press, New York, 1997).

${ }^{69}$ J. Aizpurua, P. Hanarp, D.S. Sutherland, M. Käll, G.W. Bryant, and F.J. García de Abajo, Phys. Rev. Lett. 90, 057401 (2003).

${ }^{70}$ G. Videen, D. Ngo, and M.B. Hart, Opt. Commun. 125, 275 (1996).

${ }^{71}$ V.Z. Lozovski and S.I. Bozhevolnyi, J. Phys.: Condens. Matter 14, 13597 (2002).

${ }^{72}$ A.A. Lalayan, K.S. Bagdasaryan, P.G. Petrosyan, and K.V. Nerkararyan, J. Appl. Phys. 91, 2965 (2002).

${ }^{73}$ Z. Wang, Z. Peng, K. Lu, and W. Wen, Appl. Phys. Lett. 82, 1796 (2003).

${ }^{74}$ I.P. Kozlov, Tech. Phys. Lett. 29, 272 (2003).

${ }^{75}$ J.B. Pendry, Phys. Rev. Lett. 85, 3966 (2000).

${ }^{76}$ M. Abramowitz and I. A. Stegun, Handbook of Mathematical Functions (Dover, New York, 1964).

${ }^{77}$ F. W. J. Olver, Asymptotics and Special Functions (Academic Press, New York, 1974). 